\title{
Gastric neuroendocrine tumor with extensive ossification
}

A 40-year-old man underwent endoscopic ultrasound (EUS) because of suspected submucosal gastric tumor diagnosed after an episode of severe bleeding ( $\bullet$ Fig. 1 ). EUS revealed a well-delineated, $18-\mathrm{mm}$ hypoechogenic lesion in the submucosa with a calcified area inside it ( $\bullet$ Fig. 2 , - Video 1). Three lymph nodes near the celiac trunk showed the same characteristics of the gastric lesion: well-delineated and hypoechogenic, with central calcification ( Fig.3, Video 1). A fine-needle

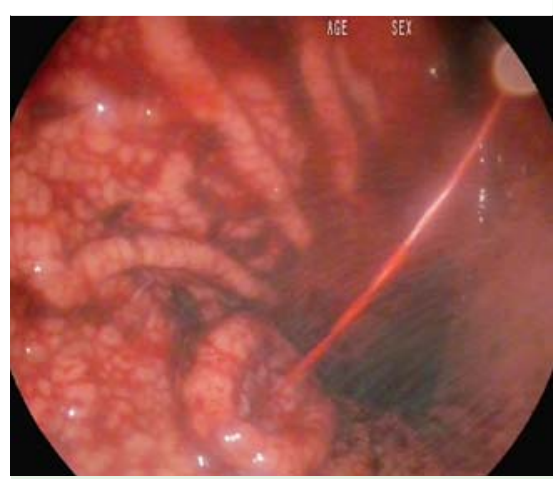

Fig. 1 Endoscopic view in a 40-year-old man showing a gastric neoplasm with active bleeding.
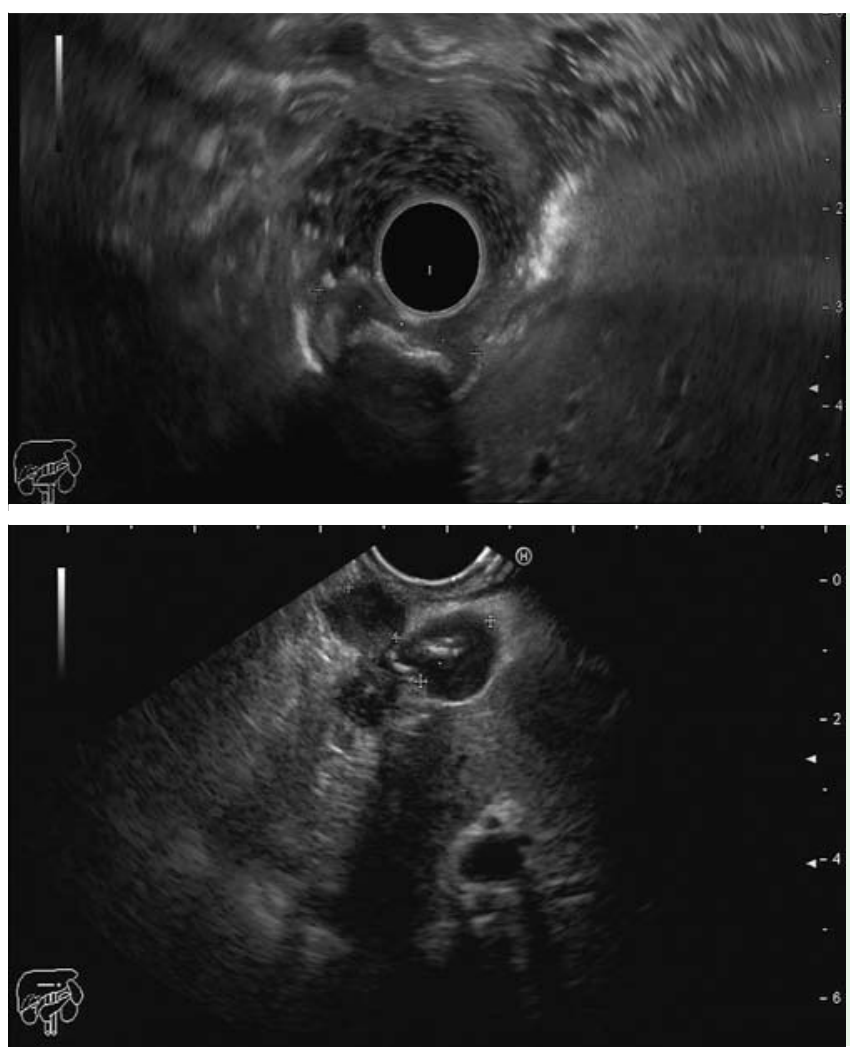

Fig. 2 Ultrasonographic image of submucosal gastric tumor.

aspiration (FNA) biopsy of the largest lymph node $(14 \mathrm{~mm})$ was carried out using a 22-G needle, and histological examination revealed reactive lymphadenitis.

The patient was referred for surgery, which showed an ulcerated submucosal tumor in the anterior gastric wall with pathological lymph nodes in the coronary gastric chain. The finding of the intraoperative study was a neuroendocrine gastric tumor ( $\bullet$ Fig.4) with nodal neuroendocrine metastasis. All the regional lymph nodes were removed and a total gastrectomy was carried out. An intraoperative ultrasound examination was done to rule out any pancreatic or duodenal lesions. Microscopically, the tumor consisted of uniform, small cells with round or oval nuclei and moderate atypia, lying in nests and within the bone trabeculae. The number of mitoses was 4 per 10 high power field (HPF). Extensive stromal bone formation was seen within the tumor ( Fig.5). The tumor cells stained positive for cytokeratin, chromogranin A ( $\bullet$ Fig.6), synaptophysin, enolase, and

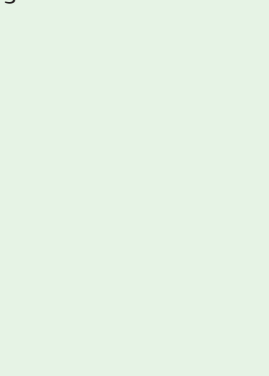

Fig. 3 The ossified lymph nodes.

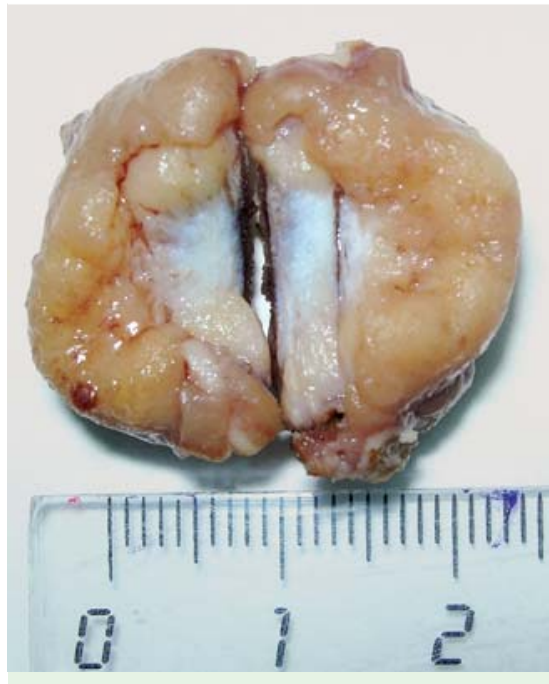

Fig. 4 Gross appearance of the gastric tumor with bone formation in the central area of tumor.

CD56, and the index of tumoral proliferation was $15 \%$. Final histopathologic diagnosis was neuroendocrine tumor G2 (World Health Organization [WHO] classification, 2010).

Most gastric neuroendocrine tumors are multiple and associated with hypergastrinemia. Solitary tumors have a poor prognosis [1]. To our knowledge, there are only four reports of gastric carcinoid tumors with ossification [2-5]. We report a case of a type 3 (G2) neuroendocrine gastric tumor with lymph node metastases with ossification diagnosed with EUS.

Endoscopy_UCTN_Code_CCL_1AB_2AD_3AB

Competing interests: None 


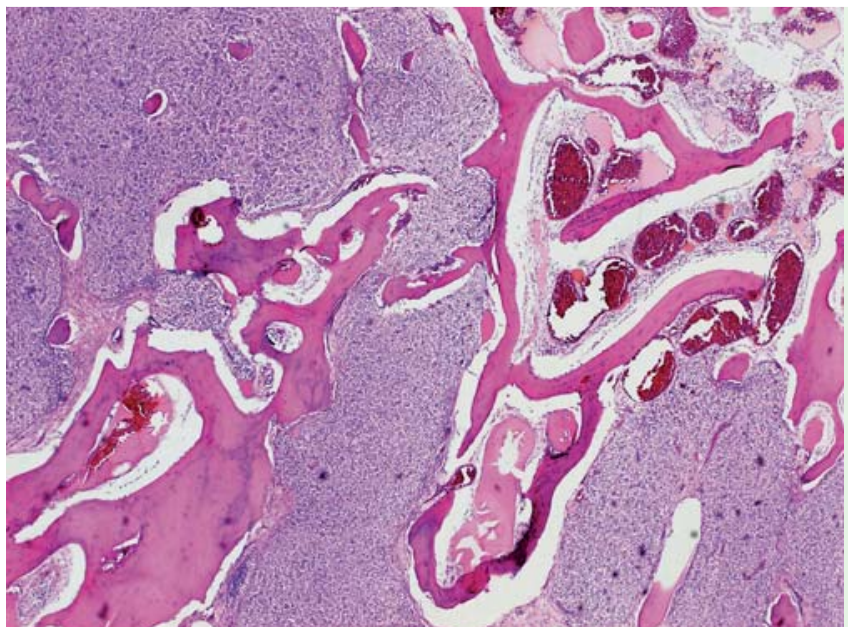

Fig. 5 Neuroendocrine tumor with extensive ossification.

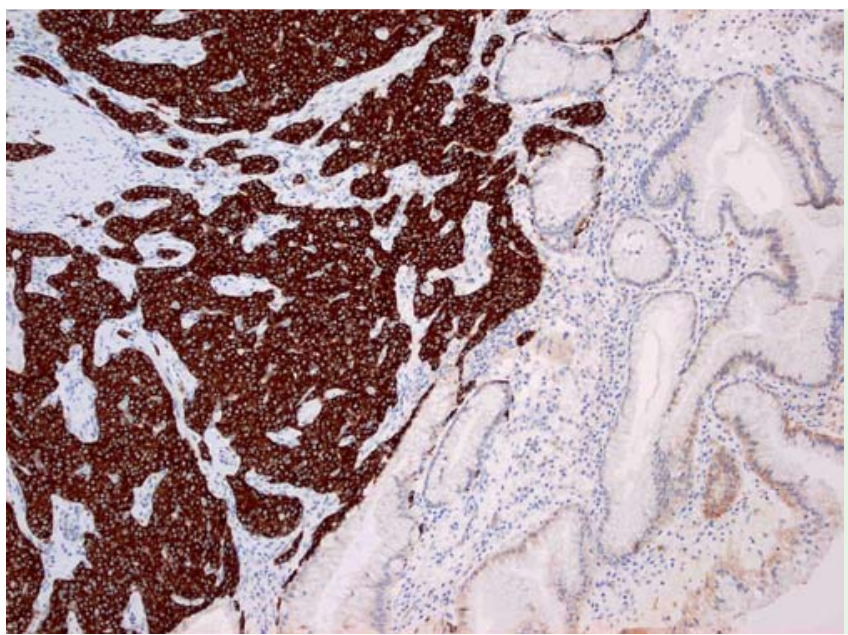

Fig. 6 Positive immunostaining for chromogranin A.

D. Del Pozo Prietoํㅜ, M. D. Vélez²,

L. Espinosa', P. González de Canales ${ }^{1}$, I. Moral ${ }^{1}$, M. Villafruela'

${ }^{1}$ S. Aparato Digestivo, Hospital Príncipe de Asturias, Alcalá de Henares, Madrid, Spain

2 S. Anatomía Patológica, Hospital Príncipe de Asturias, Alcalá de Henares, Madrid, Spain
Bibliography

DOI http://dx.doi.org/

10.1055/s-0032-1309715

Endoscopy 2012; 44: E271-E272

(c) Georg Thieme Verlag KG

Stuttgart · New York

ISSN 0013-726X

Corresponding author

\section{D. del Pozo Prieto}

S. Aparato Digestivo

H. Príncipe de Asturias

Ctra, Alcalá-Meco 28850

Alcalá de Henares

Madrid

Spain

dpozo.hupa@salud.madrid.org plasia. Arch Surg 1981; 116: 118-121

4 Linz AJ, Sheridan BJ, Carney JA. Metastatic ossified gastric carcinoid with hypergastrinemia associated with gastric and thyroid autoantibodies. J Am Osteopath Assoc 1990; 90: 1092 - 1099

5 Yamagishi SI, Suzuki T, Ohkuro H et al. Ossifying gastric carcinoid tumor containing bone morphogenetic protein, osteopontin and osteonectin. J Endocrinol Invest 2004; 27: $870-873$ 